\title{
High Quality Electron Beams from a Laser Wakefield Accelerator
}

\author{
S. M. Wiggins ${ }^{1}$, R. P. Shanks ${ }^{1}$, R. C. Issac ${ }^{1}$, G. H. Welsh ${ }^{1}$, M. P. Anania ${ }^{1}$, E. Brunetti ${ }^{1}$, G. Vieux ${ }^{1}$, \\ S. Cipiccia ${ }^{1}$, B. Ersfeld ${ }^{1}$, M. R. Islam ${ }^{1}$, R. T. L. Burgess ${ }^{1}$, G. Manahan $^{1}$, C. Aniculaesei $^{1}$, \\ W. A. Gillespie ${ }^{2}$, A. M. MacLeod ${ }^{3}$, D. A. Jaroszynski ${ }^{1}$ \\ ${ }^{1}$ SUPA, Department of Physics, University of Strathclyde, Glasgow G4 0NG, U.K. \\ ${ }^{2}$ SUPA, Division of Electronic Engineering and Physics, University of Dundee, Dundee DD1 4HN, U.K. \\ ${ }^{3}$ School of Computing and Engineering Systems, University of Abertay Dundee, Dundee DD1 1HG, U.K. \\ Email: m.wiggins@phys.strath.ac.uk
}

\begin{abstract}
Very stable, high quality electron beams (current $~ 10 \mathrm{kA}$, energy spread $<1 \%$, emittance $\sim 1 \pi \mathrm{mm} \mathrm{mrad}$ ) have been generated in a laser-plasma accelerator driven by $25 \mathrm{TW}$ femtosecond laser pulses. (C) 2010 Optical Society of America

OCIS codes: (350.4990) Particles; (350.5400) Plasmas
\end{abstract}

\section{Introduction}

The laser wakefield accelerator (LWFA) mechanism was first proposed thirty years ago by Tajima and Dawson [1], as an attractive alternative to RF accelerator technology. After three decades of research, the LWFA is now capable of producing $100 \mathrm{~s}$ of $\mathrm{MeV}$ electron bunches from mm-long gas jets and $1 \mathrm{GeV}$ electron bunches from a $33 \mathrm{~mm}$ long discharge capillary plasma waveguide [2].

The first experimental demonstrations of a LWFA as a driver of synchrotron radiation from an undulator have recently been published $[3,4]$. However, a major challenge is to improve the beam quality to a point where a freeelectron laser (FEL) becomes feasible. This requires a relative energy spread of less than $1 \%$ and normalised transverse emittance of $\sim 1 \pi \mathrm{mm}$ mrad for beams with charge $\sim 10 \mathrm{pC}$ and duration $\sim 10 \mathrm{fs}$. Generation of such beams in the $0.5-1 \mathrm{GeV}$ energy range will allow ultra-compact, very high peak brilliance sources of coherent X-ray radiation to be realised.

\section{Experimental Setup}

An experimental programme to demonstrate the LWFA as a driver of compact radiation sources is currently being conducted on the Advanced Laser-Plasma High-Energy Accelerators towards X-rays (ALPHA-X) laser-wakefield accelerator beam line at the University of Strathclyde [5]. The experimental setup is shown in Fig. 1: electrons are accelerated in a relativistically self-guiding plasma channel formed in a $\mathrm{H}_{2}$ gas jet (nozzle diameter $2 \mathrm{~mm}$, plasma density $\left.\sim 1 \times 10^{19} \mathrm{~cm}^{-3}\right)$ by a Ti:sapphire laser pulse $(\lambda=800 \mathrm{~nm}$, energy $=900 \mathrm{~mJ}$, pulse duration $=35 \mathrm{fs})$. The beam is focused just inside the leading edge of the gas jet (normalised vector potential $\mathrm{a}_{0} \approx 1$ ).

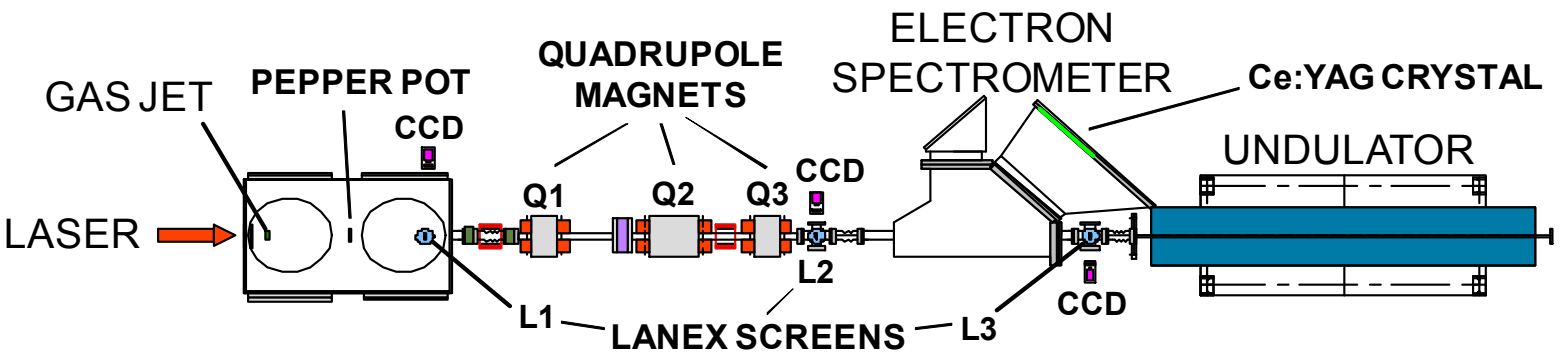

Fig. 1. Setup of the ALPHA-X wakefield accelerator beam line. Not shown is the CCD camera used to image the Ce:YAG crystal.

At these intensities electrons are self-injected from the background plasma into the plasma density wake (bubble) trailing behind the laser pulse through the combined action of the ponderomotive force of the laser and the plasma restoring force. Beams emerging from the plasma are imaged downstream using a series of pop-in Lanex scintillating screens. A pop-in tungsten pepper-pot mask is used to measure the r.m.s. transverse emittance of the beam. This consists of a $11 \times 11$ matrix of holes (mean diameter $=52 \mu \mathrm{m})$ with beamlets imaged on the first Lanex 
screen. Measurements of the electron energy spectra have been carried out using a high resolution magnetic dipole spectrometer. Scintillating Ce:YAG crystals positioned at the focal plane are used to image electrons exiting the spectrometer field and the image is captured on a 12-bit CCD camera. The transportation of the beam through the spectrometer can be optimised using quadrupole electromagnets.

\section{Experimental Results}

Imaging the electron beam profile on the first Lanex screen indicates a typical r.m.s. divergence of around 3 mrad. Figure 2(a) shows a pepper-pot image for a 4 mrad divergence beam and the resulting phase-space plot for the horizontal axis. This sets an upper limit for the normalised emittance of $(5.5 \pm 1) \pi \mathrm{mm}$ mrad, limited by the detector resolution and mask hole size. This in turn sets a maximum source size of $18 \mu \mathrm{m}$, which is obviously much larger than the bubble size and therefore unrealistic. However, GPT code simulations [6] using a more appropriate source size $(\approx 3 \mu \mathrm{m})$ indicates that a 4 mrad divergence beam would imply an emittance of the order of $0.5-1.0 \pi \mathrm{mm} \mathrm{mrad}$.
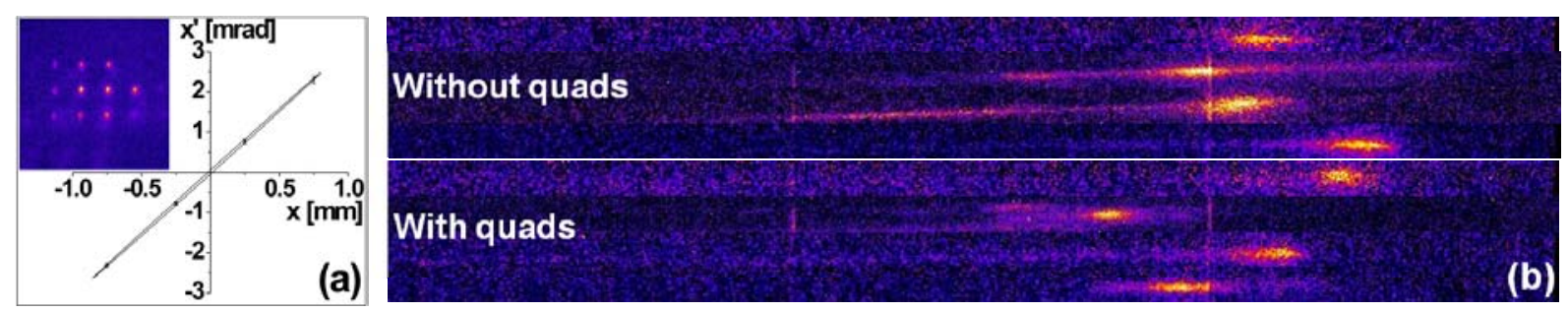

Fig. 2. (a) Phase-space diagram derived from the pepper-pot image shown in the inset and (b) eight representative electron spectra as captured on the Ce:YAG crystal when operating without and with additional quadrupole fields. The energy range shown is $60-100 \mathrm{MeV}$ (left to right).

Energy spectra have been measured using a spectrometer with a magnetic field strength of $0.59 \mathrm{~T}$. A typical spectrum consists of a single high energy peak as shown in Fig. 2(b). The average central energy is $83 \pm 5 \mathrm{MeV}$ (that is, $\pm 6 \%$ energy stability). The maximum central energy is $90 \mathrm{MeV}$. Two distinct operating modes of the spectrometer have been used to characterise the relative energy spread. Firstly, when the beam is unfocused and diverging, i.e., the quadrupole fields are turned off, the average r.m.s. relative energy spread is $\sigma_{\gamma} / \gamma=1.8 \pm 0.2 \%$. With additional quadrupole focusing, beam transportation and focusing in the spectrometer is improved. In this case, $\sigma_{\gamma} / \gamma=1.2 \pm 0.3 \%$ and the smallest value measured is $0.8 \%$. Since the quadrupole focusing is not quite optimal (as indicated by monitoring the beam profile), these measurements still represent an upper limit on the energy spread. Significantly, GPT modelling of the electron beam transport through the spectrometer shows that detection of such narrow energy spreads requires a normalised emittance $\sim 0.5 \pi \mathrm{mm}$ mrad. Excellent agreement is found between the operating modes such that both data sets indicate an actual average energy spread of $\sim 0.7-1.0 \%$, accounting for the respective spectrometer response. Furthermore, the measured energy spread dependence on charge (which is of the order of $10 \mathrm{pC})$ implies a short bunch length $(\sim 0.3 \mu \mathrm{m})$ that is consistent with simulations of our wakefield accelerator conditions obtained with a self-consistent reduced model featuring a quasi-static approximation [4] and PIC code simulations using OSIRIS.

In conclusion, characterisation of the electron beam energy spread, emittance, charge and bunch length shows that the beam quality is now sufficiently high for FEL gain to be observable in the ultra-violet spectral range and steadily approaching that required for a soft X-ray FEL.

\section{References}

[1] T. Tajima and J. M. Dawson, "Laser electron accelerator," Phys. Rev. Lett. 43, 267-270 (1979).

[2] W. P. Leemans et al., "GeV electron beams from a centimetre-scale accelerator," Nature Phys. 2, 696-699 (2006).

[3] H.-P. Schlenvoigt et al., "A compact synchrotron radiation source driven by a laser-plasma wakefield accelerator," Nature Phys. 4, 130-133 (2008).

[4] J. G. Gallacher et al., "A method of determining narrow energy spread electron beams from a laser plasma wakefield accelerator using undulator radiation," Phys. Plasmas 16, 093102 (2009).

[5] D. A. Jaroszynski et al., "Radiation sources based on laser-plasma interactions," Phil. Trans. R. Soc. A 364, 689710 (2006).

[6] S. B. van der Geer et al., "3D Space-Charge Model for GPT Simulations of High Brightness Electron Bunches," in Institute of Physics Conference Series No. 175 (Institute of Physics, Bristol, UK, 2005), pp. 101. 